\title{
Harry Potter y una lengua de serpientes: un estudio de los principales rasgos fonéticos y morfosintácticos del pársel
}

\author{
Leticia Gándara Fernández ${ }^{1}$ \\ Universidad de Extremadura, España
}

\begin{abstract}
Resumen
Este trabajo presenta un estudio del pársel, lengua creada por Francis Nolan para complementar el universo fantástico de los filmes de la saga Harry Potter. Primeramente, se estudia el tratamiento que recibe esta lengua de serpientes en las novelas con el fin de observar qué función desempeña en este argumento de ficción y cómo esto condiciona el propio proceso de construcción de este proyecto lingüístico. En segundo lugar, se exponen algunos de sus principales rasgos fonéticos y morfosintácticos. Finalmente, se muestra cómo el pársel refuerza la caracterización negativa de uno de los protagonistas $\mathrm{y}$, a su vez, ayuda a la configuración de un entramado cultural estrechamente vinculado a las serpientes.

Palabras clave: lengua artificial, lengua artística, Harry Potter, pársel, fonética.
\end{abstract}

\footnotetext{
1 Para correspondencia, dirigirse a: Leticia Gándara Fernández (leticiagf@unex.es), Departamento de Filología Hispánica y Lingüística General, Facultad de Filosofía y Letras, Avda. de las Letras, s/n, 10003 Cáceres, España (Universidad de Extremadura).
} 


\title{
HARRY POTTER AND A TONGUE OF SERPENTS: A STUDY OF THE MAIN PHONETIC AND MORPHOSYNTACTIC FEATURES OF PARSELTONGUE
}

\begin{abstract}
This work presents a study of the parsel, a language created by Francis Nolan to complement the fantastic universe of the films of the Harry Potter. First, it studies the treatment that this language of snakes receives in novels in order to observe what role it plays in this fiction argument and how this conditions the construction process of this linguistic project itself. Second, it exposed some of its main phonetic and morphosyntactic features. Finally, it shows how the parsel reinforces the negative characterization of one of the protagonists and, in turn, helps the configuration of a cultural network closely linked to snakes.
\end{abstract}

Keywords: artificial languages, artistic language, Harry Potter, pársel, phonetic.

Recibido: 07/10/19 Aceptado: $14 / 08 / 20$

\section{INTRODUCCIÓN ${ }^{2}$}

Desde la antigüedad, el sueño quimérico de una lengua artificial ha recorrido diferentes etapas de la historia, acompañado inevitablemente de una evolución interna en sus motivaciones y en sus resultados, consecuencia del marco socio-cultural de cada periodo histórico (Martínez 2016: 78). Los factores que guiaron la construcción de los primeros bocetos lingüísticos son diferentes a los motivos por los que actualmente se construyen lenguas. En el siglo XVII, las razones científicas o filológicas propiciaron la creación de esquemas lingüísticos capaces de garantizar la transparencia, la racionalidad y la univocidad en la comunicación científica (Galán 2018a: 75). El deseo de solventar las ambigüedades e imperfecciones de las lenguas naturales

2 Este trabajo se enmarca en el proyecto de investigación FFI2016-76702-P, titulado En los límites del lenguaje: diseños artificiales y ficciones comunicativas, dirigido por la Dra. Carmen Galán Rodríguez y concedido por el Ministerio de Economía y Competitividad. 
conlleva el desarrollo de lenguas lógicas, perfectas y regulares, conocidas como lenguas a priori (Couturat y Leau 1903: xxviii), cuyo uso se restringía a reducidos círculos de eruditos e intelectuales de la época (Calero 1999: 24; Calero 2010: 18). La motivación científica que domina en pleno siglo XVII dará paso en el siglo XIX a una motivación pragmática (Galán 2018a: 75). Este cambio se evidencia en la aparición de otro tipo de sistemas, denominados por Couturat y Leau (1903: xxvii) lenguas a posteriori, cuyo principal objetivo era convertirse en instrumentos de comunicación universal. Estas últimas no solo se diferencian de los proyectos anteriores en su finalidad, sino también en su método de construcción. Si bien los autores de lenguas a priori no tuvieron en cuenta las lenguas naturales para la construcción de sus sistemas, los proyectistas de mediados del XIX y principios del XX se basaron en las principales estructuras gramaticales y léxicas de los idiomas europeos más extendidos para diseñar sus esquemas lingüísticos. En estos últimos, se emplearon criterios más prácticos y realistas con el fin de facilitar el aprendizaje por parte de sus posibles hablantes.

El resultado de esta intensa actividad lingüística llevada a cabo por intelectuales (no necesariamente lingüistas) de siglos anteriores ha sido una lista interminable de proyectos diferentes pero con características comunes. Generalmente, estas lenguas artificiales se han catalogado según la clasificación propuesta por Couturat y Leau en Histoire de la langue universelle (1903) a la que nos hemos referido en líneas anteriores, que se divide en "sistemas lingüísticos a priori", sistemas "mixtos" y "lenguas a posteriori". Dicha ordenación, como los propios autores previenen, no responde a una cronología rígida o lineal, sino que en muchas ocasiones se ve alterada por proyectos que sobreviven anacrónicamente a estados ya superados o, al contario, por inventores de lenguas que con sus propuestas aventajan a sus contemporáneos, adelantándose así a la mentalidad dominante de su época (Calero 1999: 10-11). Por ello, Calero (1999: 1011) señala que resulta más apropiado hablar de "familias" de proyectos que de "etapas", dado que este último concepto parece incluir un sema de progresión cronológica.

Desde mediados del siglo XX, el interés por construir lenguas artificiales se ha reavivado gracias a las múltiples propuestas surgidas en el ámbito de la ficción literaria y cinematográfica. Estas construcciones semióticas, elaboradas con una pretensión estética, "llevan al límite las posibilidades de un lenguaje [...] en consonancia armónica con la sociedad que lo sustenta" (Galán 2017:53). Pese a que se trata de lenguas diseñadas por lingüistas con una sólida formación académica, su estudio ha pasado casi desapercibido en el área de la Lingüística. Al respecto, afirma Galán que estas lenguas se han considerado despectivamente 
como el producto de una fantasía desbordada de ciertos lunáticos; y su estudio, aun siendo riguroso, o es objeto de burla académica, o no pasa de ser una "curiosidad lingüística", obviando que estas lenguas son la consecuencia de los trazaos ideológicos e históricos de cada época (2008b: 14).

De la escasez de trabajos científicos en este campo de estudio se deriva la inexistencia de una terminología precisa para denominar estos sistemas. Algunos de las expresiones más utilizadas son: "lenguas artísticas", "lenguas ficcionales", "lenguas ficticias", "ideolenguas", "lenguas construidas" o "conlang". Este último, resultado de la suma de las dos primeras sílabas de las palabras constructed y language del inglés, goza de una gran aceptación por parte de los estudiosos y seguidores de estas lenguas. Pues, en la actualidad, uno de los mayores logros de estas construcciones semióticas ha sido el de extender este fenómeno lingüístico entre el gran público (Martínez 2016: 78).

Estos proyectos diseñados fundamentalmente para satisfacer las necesidades lingüísticas y estéticas de universos fantásticos e imaginarios, poseen también un gran valor en la configuración de identidades individuales o grupales en estos mundos de ficción (Barnes y Heerden 2006: 115). Lejos de ser simples curiosidades lingüísticas, constituyen una oportunidad magnífica para llevar al límite las posibilidades del lenguaje (Galán 2017: 53) y para analizar y comprender mejor el funcionamiento y la estructura de las lenguas naturales.

En el ámbito literario, por ejemplo, las creaciones lingüísticas de $E l$ señor de los anillos (1954-5) permiten la configuración de un universo fantástico en el que conviven elfos, hobbits, hombres, enanos, orcos y otras criaturas extraordinarias. J. R. R. Tolkien (1892-1973) considera su invención lingüística un arte -"Arte Nuevo" o "Nuevo Juego"-, un actividad privada emprendida para conferirle expresión a su "estética" lingüística personal y sus fluctuaciones (Tolkien 1993). Con sus lenguas -con el quenya y el sindarin, especialmente-no solo consigue representar los ideales de belleza

3 El término conlang aparece por primera vez el 29 de julio de 1991 para denominar la lista de correo electrónico en la que se debatían cuestiones relacionadas con la creación de lenguas. Alrededor de este neologismo se crearon otros vocablos, tales como artlang, loglang, engelang, auxlang jokelang, etc. Para traducir el término conlang al español, Alex Condori propuso en el año 2000 la expresión ideolengua, también como título de una lista de distribución. Sin embargo, aunque actualmente se aceptan las dos acepciones, conlang continúa siendo la opción más utilizada, también en la lengua española. Esta ha sido aceptada por el Oxford English Dictionary y el Cambridge Dictionary. 
lingüística -o de fealdad en el caso de la lengua negra (Shippeys 2000: xiv)-, sino también construir diferentes instrumentos de comunicación que conviven en consonancia armónica con las culturas que los sustentan (Galán 2017: 53). Esta relación entre mitología y lenguaje y, en consecuencia, entre lengua y cultura recuerda el interés de autores de principios del XX (Huxley en Un mundo feliz (1932) u Orwell en 1984 (1949), entre otros) de probar en sus novelas (distópicas) la teoría del relativismo lingüístico de Sapir-Whorf ${ }^{4}$. Un buen ejemplo de una lengua diseñada para comprobar esta teoría es el láadan (literalmente, 'lengua del conocimiento perceptivo') que se incluye en la trilogía de ciencia ficción Native Tongue (1984-94) de Suzette Haden Elgin (Gándara 2016). Con esta lengua de mujeres, Elgin pretende crear una correspondencia específica entre lengua y sensibilidad femenina con el fin de modificar los límites del lenguaje y, por consiguiente, los límites del mundo y de la propia conciencia (Galán 2007: 123).

En el cine, las lenguas inventadas pretenden satisfacer las necesidades lingüísticas y estéticas de universos fantásticos e irreales; por lo que suelen ser tan insólitas y sorprendentes como las asombrosas y extrañas culturas a las que complementan. Este hecho permite, según Lozano (citado en Robles 2016), "potenciar la identificación del espectador con lo atractivo y lo exótico de razas, culturas y civilizaciones que parecen tan reales como la suya propia". El klingon de Star Trek (elaborada por Okrand en 1979) el na'vi (diseñada por Frommer en 2009) de Avatar $^{6}$, el pársel (inventado por Nolan entre 2002 y 2011) de Harry Potter o el dothraki y el valyrio (construidos por Peterson en 2011) de Juego de tronos constituyen los ejemplos más representativos de este ámbito. No todas presentan el mismo

\footnotetext{
4 Tolkien conocía a la perfección los trabajos de Sapir, Jespersen y Bloomfield, como refiere en sus tres artículos de revisión en "Philology: General Works", que escribió para The Year's Work in English Studies, volúmenes 4-6 (1925-1927).

5 El propósito de Okrand no era otro que crear un idioma cuyo sonido fuera diferente a todas las lenguas habladas en la tierra. Es probable, por tanto, que este fuera el motivo por el que la lengua base su fonética en sonidos guturales que a su vez son muy difíciles de pronunciar y entender. Gran parte de su léxico refleja aspectos relacionados directa e indirectamente con la guerra. Su complejidad léxica, junto a otros aspectos como su difícil pronunciación, pueden hacer que aprender klingon se convierta en una tarea fatigosa. La solución a este problema de aprendizaje nos la ofrece el Instituto de Lengua Klingon, así como la gran cantidad de materiales que podemos encontrar en Internet. Para mayor información, véase (Gándara 2019).

${ }^{6}$ El na'vi fue creado por el lingüista Paul. R. Frommer para la película Avatar. De nuevo, el objetivo era conseguir una lengua que fuera fácil de pronunciar para los autores pero totalmente diferente de las lenguas habladas en la Tierra. Una antítesis en toda regla, un problema difícil de resolver, el perseguir una lengua bella a la vez que extraña, un interesante reto al que no solo se enfrenta su creador sino también los actores que deben aprenderla.
} 
grado de complejidad ni todas han sido objeto de estudios científicos. La información sobre la gramática y el léxico del klingon y el dothraki, por ejemplo, se recoge en publicaciones como The Klingon Dictionary (1992) de Okrand o Living Language Dothraki (2014) de Peterson, respectivamente. Sobre el na'vi hay información actualizada en varios portales y páginas web del autor (en https://naviteri.org) o de sus seguidores (en $<$ https://learnnavi. org/>). Sin embargo, resulta complicado encontrar datos fiables sobre el pársel. Por ello, en este estudio ofrecemos un análisis de los principales rasgos fonéticos y morfosintácticos de esta lengua de serpientes. Para ello, trazamos primeramente una aproximación al proceso de elaboración de este esquema lingüístico y a cómo se incluye en el argumento de ficción de la saga Harry Potter ${ }^{7}$.

\section{EL PÁRSEL EN LA SAGA HARRY POTTER}

El pársel o 'lengua de serpientes' aparece en la saga de literatura fantástica Harry Potter de la escritora británica Joanne Kathleen Rowling (1965). Esta se basa en las aventuras de Potter, un joven aprendiz de mago que ingresa en la escuela de magia Hogwarts, en la que se enfrenta al malvado Lord Voldemort. Una de sus cualidades es su capacidad de hablar pársel, la lengua de las serpientes, que traspasa inconscientemente a Harry la noche en la que intentó matarle. Por ello, el pársel se convierte en uno de los elementos esenciales de la trama, especialmente en algunas de las novelas.

Sin embargo, a diferencia de otros sistemas lingüísticos artificiales como el quenya y el sindarin o el láadan, Rowling no desarrolló los rasgos gramaticales y léxicos del pársel para su inclusión en las novelas. Los encuentros entre Potter y las serpientes u criaturas similares se describen en lengua inglesa. Por lo tanto, no hay ninguna muestra de pársel en las novelas, a diferencia de lo que sucede en las adaptaciones cinematográficas, en las que sí es posible escuchar diálogos en esta lengua. En concreto, aparece en Harry

\footnotetext{
Conviene tener en cuenta que resulta cuanto menos difícil asumir el estudio de este sistema lingüístico, dado que no existen trabajos de investigación anteriores que se ocupen de este asunto. De hecho, para la elaboración de artículo se ha tomado como referencia el material cedido por el propio autor de la lengua, así como las entrevistas que la autora de este estudio mantuvo con Nolan durante una estancia de investigación en la Universidad de Cambridge.
} 
Potter y la cámara secreta (2002), Harry Potter y el cáliz de fuego (2005) y las dos partes de Harry Potter y las Reliquias de la muerte (2010, 2011), en las que esta lengua de reptiles tiene una función importante en la trama.

En la saga Harry Potter, la lengua pársel es hablada por serpientes y otras criaturas mágicas de esta misma familia, como los runespoor (serpiente de tres cabezas) o los basiliscos, pero también por aquellos personajes que tienen la capacidad de comunicarse con estos animales. En las novelas, el sonido de esta lengua se define de la siguiente forma:

-Hablaste en lengua pársel -le dijo Ron-, la lengua de las serpientes. Podías haber dicho cualquier cosa. No te sorprenda que Justin se asustara, parecía como si estuvieras incitando a la serpiente, o algo así. Fue escalofriante (Rowling 1999: 170).

La habilidad de hablar pársel es poco frecuente y se manifiesta como una cualidad hereditaria, que se encuentra tan solo al alcance de unos pocos. En la saga, casi todos los personajes que entienden esta lengua son descendientes de Salazar Slytherin, uno de los cuatro fundadores del Colegio Hogwarts, cuyo emblema es precisamente una serpiente. Tan solo el propio Harry y Herpo el Loco (un antiguo mago oscuro al que se atribuye la creación del primer basilisco, al que consiguió dominar a través de la lengua pársel y al que después convirtió en un Horrocrux) son capaces de comunicarse en este código sin haberlo heredado.

En las novelas, el pársel se presenta como un atributo propio de los Magos Tenebrosos. Su descubrimiento se atribuye a Paracelso, un alquimista secreto del que se sabe muy poco. Este personaje solo aparece en uno de los cromos de las ranas de chocolate que Harry recibe en su primer viaje a Hogwarts y en un busto de los pasillos de esta misma escuela. Según este, la lengua pársel dataría del siglo XVI, a pesar de que ya existiesen hablantes en tiempos más antiguos, como el propio Salazar Slytherin.

En un principio, Harry Potter no entiende por qué habla pársel, una lengua que ni tan siquiera conoce: "¿Hablé en otra lengua? Pero no comprendo... ¿Cómo puedo hablar en una lengua sin saber que la conozco?" (1998: 139). Será otro de los personajes principales, su amiga Hermione Granger, quien le descubra la importancia de hablar en una lengua que ha estado siempre relacionada con la temida Magia Oscura:

- ¿Me quieres decir qué hay de malo en impedir que una serpiente grande y asquerosa arranque a Justin la cabeza de un mordisco? -preguntó- ¿Qué importa cómo lo hice si evité que Justin tuviera que ingresar en el Club de Cazadores Sin Cabeza? 
-Sí importa - dijo Hermione, hablando por fin, en un susurro-, porque Salazar Slytherin era famoso por su capacidad de hablar con las serpientes. Por eso el símbolo de la casa de Slytherin es una serpiente (Rowling 1998: 171).

Más tarde, Harry averiguará que su dominio del pársel se encuentra estrechamente relacionado con la noche en la que Lord Voldemort intentó matarle, pues este le cedió inconscientemente algunas de sus habilidades, además de una parte de su alma. Se supone que cuando esta última desaparece del cuerpo de Harry, este pierde su habilidad de hablar en pársel. Sin embargo, en Harry Potter y el legado maldito, el protagonista vuelve a utilizar esta lengua para abrir la habitación encantada de Delphini, la supuesta hija de Voldemort.

En Harry Potter y la piedra filosofal, no aparece ninguna referencia al nombre de la lengua que emplea el protagonista para comunicarse con la serpiente a la que libera inconscientemente en el museo. Sin embargo, en Harry Potter y la cámara secreta, Rowling utiliza por primera vez el término pársel para referirse a esta desconocida lengua; un hecho justificado si tenemos en cuenta el gran peso argumental que tiene en esta segunda novela. En primer lugar, Harry comprende que es capaz de hablar una lengua que ni siquiera conoce y con la que puede comunicarse con las serpientes, una habilidad relacionada con la Magia Oscura, que no es en absoluto algo bueno, como él había pensado en un principio. La capacidad de entender pársel le permite oír voces que nadie escucha en los pasillos de Hogwarts, pero también levantará ciertas sospechas sobre si Harry es el verdadero heredero de Salazar Slytherin; pues solamente el fundador de la Casa Slytherin poseía la habilidad de hablar pársel. Finalmente, Harry se verá obligado a utilizar el pársel para abrir la cámara secreta, en la que enfrenta al enorme basilisco dominado por Lord Voldemort:

-Ron -musitó-. ¡Esto es! Aquí está la respuesta. El monstruo de la cámara es un basilisco, juna serpiente gigante! Por eso he oído a veces esa voz por todo el colegio, y nadie más la ha oído: porque yo comprendo la lengua pársel... (Rowling 2001: 246).

La lengua pársel reaparece en Harry Potter y el cáliz de fuego. Desde un principio, Voldemort se encuentra siempre acompañado por su fiel compañera Nagini, una enorme serpiente que lo alimenta y sobre la que posee un control absoluto. Con esta, el malvado mantendría supuestas conversaciones en 
lengua pársel, que se describen de la siguiente manera en la obra desde la perspectiva de un muggle

Antes de que hubiera tomado una decisión, la serpiente había llegado al punto del corredor en que él se encontraba e, increíble, milagrosamente, pasó de largo; iba siguiendo los sonidos siseantes, como escupitajos, que emitía la voz al otro lado de la puerta y, al cabo de unos segundos, la punta de su cola adornada con rombos había desaparecido por el resquicio de la puerta.

Frank tenía la frente empapada en sudor, y la mano con que sostenía el cayado le temblaba. Dentro de la habitación, la fría voz seguía silbando, y a Frank se le ocurrió una idea extraña, una idea imposible: que aquel hombre era capaz de hablar con las serpientes (Rowling 2001: 19).

Con todo, en el relato encontramos también ciertas descripciones en las que se detalla cómo la fisionomía de Voldemort es también similar a la de una serpiente:

El hombre delgado salió del caldero, mirando a Harry fijamente... y Harry contempló el rostro que había nutrido sus pesadillas durante los últimos tres años. Más blanco que una calavera, con ojos de un rojo amoratado, y la nariz tan aplastada como la de una serpiente, con pequeñas rajas en ella en vez de orificios.

Lord Voldemort había vuelto (Rowling 2001: 560).

Este parecido razonable entre Voldemort y una serpiente bien podría relacionarse con uno de los poderes que aún conserva el malvado y que le permite mantener viva su alma: "el de ocupar los cuerpos de otros" (Rowling 2001: 569). Voldemort logró sobrevivir durante años gracias a su permanencia en cuerpos de animales, especialmente de serpientes, aunque en ellos, como afirma, "no estaba mucho mejor que siendo puro espíritu" (Rowling 2001: 569). Las razones obedecían a su incapacidad de hacer magia desde estos cuerpos y a que su posesión les acortaba la vida. En consecuencia, Harry Potter y el cáliz de fuego alberga un sinfín de elementos relacionados con el mundo de las serpientes que contribuyen a la configuración del personaje de Lord Voldemort pero que, a su vez, esconden un complejo entramado de significaciones ocultas provenientes de diferentes

\footnotetext{
8 En el Cambridge Dictionary online, un muggle es "a person who does not have a particular type of skill or knowledge"; por extensión metafórica, Rowling aplica el término a aquellas personas que no poseen poderes mágicos.
} 
culturas y tradiciones, en las que parece haberse inspirado la autora para escribir la saga. Resulta difícil pensar que es pura coincidencia esa especie de reencarnación de Voldemort en cuerpos de serpientes, si tenemos en cuenta que en la tradición cristiana también Satanás se valió de este animal para incitar a Eva a comer de la fruta prohibida en el Paraíso. Por otro lado, tampoco es arbitraria la elección del nombre Nagini para la serpiente aliada de Voldemort. Dicho término recuerda a la palabra naguini (del sánscrito $n \bar{a} g a$, 'serpiente'), que hace referencia a una deidad femenina con forma de serpiente. En las mitologías hinduista y budista, las nagas suelen ser representadas como criaturas malvadas, en constante conflicto con Garudá, una gigantesca águila con forma antropomórfica .

En Harry Potter y las Reliquias de la Muerte, el pársel volverá a ocupar un lugar especial en el desarrollo de la trama, no solo por la presencia de serpientes en el argumento, sino también por la importancia que esta tiene en algunas escenas de la obra. Además de los numerosos pasajes en los que se asocia el rostro de Voldemort con el de una serpiente, también aparecen momentos en las que este habla con su fiel aliada Nagini, que sobrevive hasta el final de la obra por contener una parte del alma del malvado.

\section{UN ESTUDIO DE LOS PRINCIPALES RASGOS FONÉTICOS Y MORFOSINTÁCTICOS DEL PÁRSEL}

\subsection{EL TÉRMINO PÁRSEL}

El término pársel fue utilizado originariamente para referirse a una persona que tenía problemas en el labio, algo semejante a un labio leporino. Teniendo

9 En esta línea argumental, conviene recordar la importancia de Fawkes, supuesta mascota de Dumbledore, en el enfrentamiento entre Harry y el basilisco en la Cámara de los Secretos. Se trata de un fénix, cuyas lágrimas poseen propiedades curativas y del que fueron tomadas las plumas que componen las varitas mágicas de Voldemort y Harry. En la mitología, el fénix es el símbolo de la inmortalidad, aquella que Voldemort ansiaba conseguir. Sin embargo, dicho término es también un juego de palabras, que hace referencia al nombre de Guy Fawkes, líder de la fallida Conspiración de la Pólvora que tuvo lugar en Gran Bretaña el 5 de noviembre de 1605 para destruir la sede del Parlamento en Londres. Desde esa fecha, cada 5 de noviembre se conmemora esta festividad en la noche de las hogueras. En consecuencia, el uso de este nombre para la mascota de Dumbledore relaciona dos historias a través del mito del fénix que se prende en llamas cuando cree que ha llegado su hora, renace después de sus cenizas y vive para siempre. 
en cuenta la fisonomía de Lord Voldemort, personaje al que se asocia la lengua, no es extraño pensar que esta acepción rondaba en la mente de la autora cuando escribió sus novelas ${ }^{10}$. Sin embargo, otros investigadores han apuntado diferentes interpretaciones de la palabra "pársel". Gizelle L. Anatol establece ciertas conexiones del término Parseltongue con la palabra Parsi, el nombre de una comunidad religiosa que habita al oeste de la India, especialmente en Bombay. Y añade:

For British readers, references to Parsees might unconsciously evoke images of fakirs and other mystics who perform feats of magic and endurance, such as charming snakes from baskets and walking on coals. [...] snakes in the Harry Potter books symbolize inherent femaleness, seductiveness, duplicity, sexual excess, sulking silence, and above all, danger (2003: 169-173).

Por otro lado, este término puede también interpretarse como un neologismo morfológico creado por la autora para satisfacer las necesidades argumentales de la novela. Hilsheimer, por su parte, señala que:

The first part of the word, parsel, likely comes from the French verb, parter, which means -to speak. This would make sense in context as this is a spoken language. It is possible that the second half of the word, tongue can mean language, as in mother tongue or quite, literally, a tongue (2011: 20).

Aunque Rowling elige el término parselmouth para referirse a esta lengua de serpientes en las novelas, no desarrolla sus rasgos fonéticos, gramaticales y léxicos. Fue Francis Nolan, profesor de la Universidad de Cambridge, el encargado de desarrollar el pársel para su aparición en las adaptaciones cinematográficas de Harry Potter ${ }^{11}$. A diferencia de otros inventores de lenguas artificiales, Nolan no desarrolló una lengua completa; tan solo generó una serie de rasgos fonéticos y gramaticales que le permitiesen traducir los diálogos que aparecen en los filmes. Chris Columbus, director de la segunda

10 En una entrevista de 2003, Rowling define el término parselmouth de la siguiente forma: "Parselmouth is an old word for someone who has a problem with the mouth, like a hare lip".

11 Francis Nolan es profesor en la Universidad de Cambridge (Phonetics Laboratory, Department of Theoretical and Applied Linguistics). Sus intereses se centran especialmente en el campo de la fonética: características de los hablantes, prosodia (melodía y ritmo del discurso), teoría fonética y aplicaciones de la fonética. 
película, encargó a Nolan la ardua tarea de elaborar las características lingüísticas que conformarían el pársel.

En el diseño de la lengua, la intención de Nolan no fue otra que la de crear una lengua cuyo sonido fuese semejante al que emiten las serpientes, diferente de las lenguas naturales y "fácil" de pronunciar para los actores. Desde un primer momento, el autor optó por la traducción de las expresiones que aparecen en los filmes más que por la elaboración de una lengua completa. Por ello, no puede considerarse una lengua artificial como tal, pues las únicas manifestaciones existentes en pársel son las oraciones que aparecen en las películas. En este sentido, algunos estudiosos y apasionados por los conlangs incluyen al pársel en un cajón de sastre al que han etiquetado con el nombre de relex, ya que no puede considerarse una "lengua completa", pero se crea mediante el uso de reglas lingüísticas reales (Olivera 2015: 58) ${ }^{12}$.

Nolan construye este código lingüístico conforme a los siguientes principios: 1) debía emular los silbidos que emiten las serpientes; 2) tenía que ser apropiado y "fácil" de pronunciar por los actores y 3) pretendía provocar cierta desconfianza y terror al ser escuchado. Para la creación de un sistema de tales características, Nolan decide combinar su escaso saber sobre serpientes con sus amplios conocimientos de fonética. Por lo que para la elaboración del pársel, Nolan toma como referencia tanto lenguas naturales como lenguas inventadas. De las primeras, son notables las influencias del galés, el euskera o el estonio, mientras que, de las segundas, destaca el Ithkuil. Este sistema fue inventado por John Quijada entre 1978 y 2004 con el objetivo de acabar con las imprecisiones semánticas que engloban las lenguas naturales. Según Nolan, se trata de un sistema "designed to express deeper levels of human cognition overtly and clearly...yet briefly" (2015).

Con respecto a la fonética, Nolan estudió las variaciones sonoras presentes en diferentes lenguas del mundo. Consonantes y vocales aparecen de forma desigual en los distintos idiomas y se combinan de diferente manera en cada sistema lingüístico. Esta divergencia se manifiesta en casos como el adigué (Cáucaso), con 50 o 60 consonantes dependiendo del análisis y del dialecto, y 3 vocales, en contraste con lo que sucede en las lenguas germánicas, que

12 El término relex es un acortamiento de relexificación o proceso de sustitución del vocabulario de una lengua. En el contexto de las lenguas artificiales, este se utiliza en casos en los que se mantiene la gramática de una lengua natural, pero se sustituye su vocabulario por uno de creación propia. En el caso del pársel, al tratarse de un código basado principalmente en la fonética y que apenas presenta léxico, conviene matizar que su inclusión en este grupo se debe al uso de principios fonéticos y fonológicos propios de las lenguas naturales, más que a la sustitución de términos de las lenguas naturales por otros inventados. 
presentan un número promedio de ambas. Por otro lado, observamos otras como el !xóõ (Botsuana y algunas zonas de Namibia) con 141 fonemas en total, 95 consonánticos y 46 vocálicos (incluidos los diptongos), y el hawaiano con 8 fonemas consonánticos y 5 vocálicos.

\subsection{FONÉTICA Y FONOLOGÍA}

El pársel presenta tanto sonidos vocálicos como consonánticos. En cuanto a los primeros, encontramos tan solo tres vocales $(a, e, i)$, con un gran predominio de la vocal abierta $a$. De ellas, destacamos la $[\varepsilon]$, vocal semiabierta anterior no redondeada y la [I], vocal casi cerrada anterior no redondeada ${ }^{13}$.

Las consonantes son mayoritariamente fricativas; entre las que destacan la fricativa dental sorda $/ \theta /$ (representa th en inglés), la fricativa postalveolar sorda [S] y, especialmente, la fricativa velar sorda $/ \mathrm{s} /$. Esta última puede aparecer incluso duplicada o triplicada. Este hecho se debe a que en las fricativas el aire no sufre una obstrucción total, sino una fuerte turbulencia debido al estrechamiento de los órganos articulatorios. Por consiguiente, podemos pronunciarlas seguidamente hasta quedarnos sin respiración. Si probamos, por ejemplo, a pronunciar "ssss" ininterrumpidamente, conseguiremos un sonido semejante al que emiten las serpientes; este era el propósito de Nolan al utilizar estos fonemas. También destacan la fricativa lateral alveolar sorda $/ \mathrm{t} / \mathrm{y}$ la fricativa glotal sorda $/ \mathrm{h} /$. La primera es una influencia directa del galés y se registra en términos como llath ("yard" $=36$ ), cuya transcripción es /a: $\theta /$. En cuanto a la segunda, se articula desde la glotis y se trata de un sonido similar al que se encuentra en caixa en portugués y shower en inglés; en pársel lo encontramos en palabras como ['sjehh], en la que aparece en posición final de sílaba y como geminada.

Los sonidos faríngeos aparecen con frecuencia en pársel. También reciben la denominación de "consonantes enfáticas" y se producen aproximando la raíz de la lengua a la pared faríngea, situada entre el velo del paladar y la

13 En la pronunciación de $[\varepsilon]$, la lengua se sitúa a medias entre una vocal abierta y una cerrada; en concreto, tan adelante como sea posible en la boca sin crear una constricción que se pueda calificar como consonante. Finalmente, lo más interesante, y por lo que posiblemente Nolan eligió este sonido, es que no se trata de un sonido vocálico redondeado, por lo que los labios no se abocinan. Este es un rasgo que comparte con el sonido vocálico [I], en la que la lengua se contrae menos que al formar una vocal cerrada, posicionándose ligeramente más recogida que en una vocal anterior (Wikipedia). 
laringe. En ese punto, se produce una constricción que recuerda a la estrechez de una serpiente, como se aprecia en la siguiente figura:

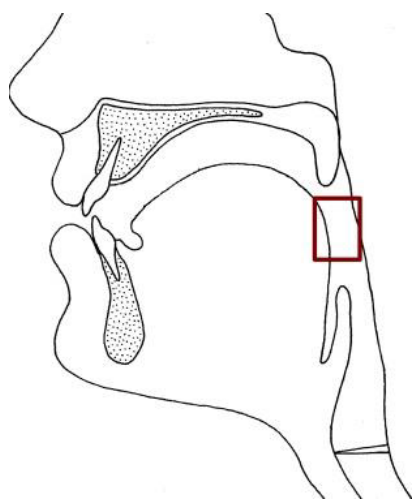

Ilustración 1. Faríngea (Hewleet y Beck, 2006)

Los sonidos faríngeos son muy frecuentes en las lenguas árabes, aunque también se encuentran en otros idiomas como el gallego, el portugués o el danés. Según el Alfabeto Fonético Internacional (AFI), se incluyen entre las fricativas y se dividen en la fricativa faríngea sonora $[\mathrm{C}]$ y la fricativa faríngea sorda [ $\hbar$. A diferencia de esta última, de la que no podemos garantizar su presencia en pársel, la primera sí aparece en esta lengua de serpientes. Este hecho se debe a que, según Nolan ${ }^{14}$ (2015), estos se perciben como sonidos amenazadores para todo aquel hablante que no los tenga en su lengua. El autor destaca algunos de los rasgos distintivos del pársel en el siguiente espectrograma ${ }^{15}$ :

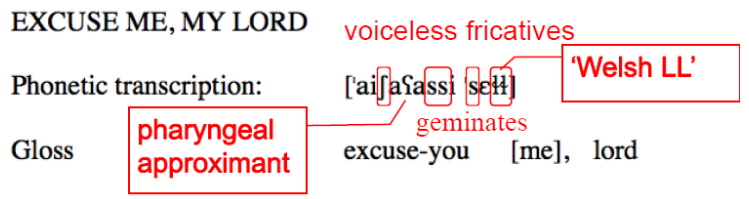

Ilustración 2. Ejemplo en pársel (Nolan 2015).

14 Material correspondiente a una conferencia en Kent que el profesor Nolan cedió a la autora del trabajo en una entrevista personal en 2015.

15 El espectrograma representa las variaciones de la frecuencia-eje vertical-y la amplitud -nivel de grises- de la señal sonora a lo largo del tiempo - eje horizontal-. Nos permite analizar la sonoridad, la duración, la estructura formántica (timbre), la amplitud (intensidad), las pausas, el acento, la velocidad de elocución y el ritmo (Llisterri 2018). 


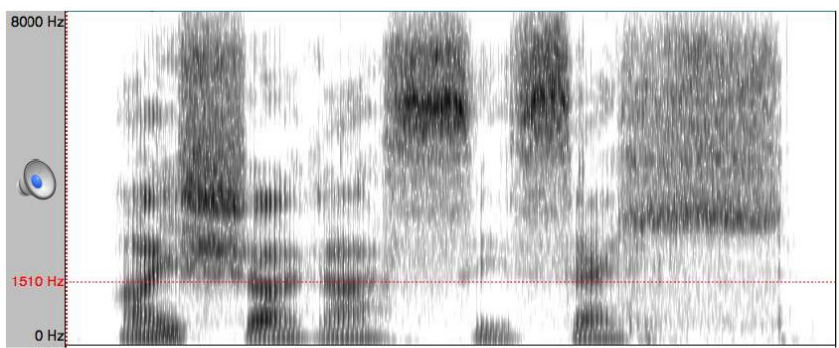

'aI $\int$ a $\&$ a s s i 's $\varepsilon$ \&

Ilustración 3. Espectrograma del enunciado "excuseyou [me], lord" (Nolan 2015)

En la transcripción de este enunciado, destacan a su vez dos rasgos fundamentales: la presencia de diptongos crecientes y las consonantes dobles o geminadas. En cuanto a los primeros, conviene destacar la aparición de [ai] o [je] en ejemplos como ['aifaSassi'seH1] y ['sjehh].

Así mismo, con el fin de obtener un mayor número de silbidos en pársel, Nolan incluye consonantes dobles o geminadas. En este caso, toma como referencia lenguas como el finés o el estonio. Esta última presenta tres grados de extensión: fonemas breves, largos y extralargos. Estos no se distinguen ortográficamente pero sí producen oposiciones semánticas, como se advierte en el siguiente ejemplo ${ }^{16}$.

\begin{tabular}{|l|l|l|}
\hline kasi & kassi & kassi \\
\hline$[$ kasi] & {$[$ kassi } & {$[$ kasssi } \\
\hline clear [off] & cat (gen) & cat (partitive) \\
\hline
\end{tabular}

Tabla 1. Grados de extensión del término kasi

La aparición de este rasgo en las palabras en pársel conlleva un alargamiento de la pronunciación, ya que este es uno de los objetivos que se marcó

16 Algunas lenguas presentan una oposición semántica creada por la duración de los sonidos. El finés es un ejemplo de lengua en la que la cantidad desempeña una función lingüística distintiva. En dicha lengua, se utiliza profusamente la diferencia entre sonidos cortos y largos para distinguir palabras. Los sonidos largos pueden aparecer en casi cualquier posición de palabra. Como ejemplos, citamos casos como korpi (bosque) y korppi (cuervo) o kansa (pueblo) y kanssa (con) (Karlsson 1991: 27-28). 
Nolan en su invención lingüística. Sin embargo, no conviene confundir las consonantes geminadas con dígrafos como el galés - $l l-$, que aparecen, por ejemplo, en términos como [scłt] 'señor'. También es fundamental la aparición de la velar nasal [ $\mathrm{y}]$. Este sonido suele aparecer ante consonantes oclusivas velares como $/ \mathrm{k} / \mathrm{y} / \mathrm{g} /$ en español, o en algunos dialectos como el peruano ribereño en posición final de sílaba, como en [ra'son]. Sin embargo, en pársel aparece en posición inicial de sílaba, como en ['yaPalas]. En este caso, observamos también la presencia de la oclusiva glotal sorda [?]. Finalmente, el pársel no contiene sonidos bilabiales, tales como como /b/, $/ \mathrm{p} / \mathrm{y} / \mathrm{m} /$, dado que las serpientes no tienen labios y no les sería posible producirlos. Finalmente, el efecto deseado al producir oralmente este sistema lingüístico se consigue por medio del fenómeno prosódico del susurro o pronunciación parentética en voz baja. Por tanto, este se convierte en uno de los elementos fundamentales en la construcción de este proyecto lingüístico.

\subsection{MORFosintaXis}

En primer lugar, conviene destacar que el orden de palabras utilizado en el pársel es de los menos habituales entre las lenguas naturales. La disposición Verbo Sujeto Objeto (VSO), propia del árabe, el tágalo, el galés o el irlandés (esta última mencionada por Nolan en sus conferencias), sirve al autor para dotar de una mayor extrañeza a su proyecto lingüístico. En el ejemplo que mostramos a continuación, se observa cómo is constituye el verbo, muggle, el sujeto y door-of in front, el objeto (ejemplo proporcionado por Nolan en 2015):

THERE IS A MUGGLE OUTSIDE THE DOOR, LORD

$\begin{array}{llll}\text { Phonetic transcription: } & \text { [sa'ya?alas } & \text { 'nessja 'leha } & \text { sełl]. } \\ \text { Gloss: } & \text { is muggle } & \text { door-of in front, } & \text { lord }\end{array}$

En algunas lenguas, el orden de palabras es determinante a la hora de expresar la función gramatical que cumple una palabra en una oración. En inglés, por ejemplo, The cat loves the girl no significa lo mismo que The girl loves the cat. En el primer caso, es el gato el que quiere a la chica, mientras que, en el segundo, es la chica la que quiere al gato. Conocemos quién muestra dicho sentimiento por la posición que ocupan dichos términos en el enunciado. Sin embargo, en otras lenguas, dicha función se expresa también a través de un sistema de casos. Aunque en pársel no aparece de forma explícita dicho sistema, Nolan mencionó su existencia. Algo parecido sucede con las posposiciones, de las que sí conservamos un ejemplo; este aparece 
en la oración expuesta en el gráfico anterior: ['leha] (in front). Conviene destacar que las posposiciones suelen asociarse a lenguas del tipo SOV, mientras que las preposiciones se vinculan a los modelos SVO y VSO. Pese a no presentar el orden SOV, en pársel, la aparición de posposiciones se encuentra justificada por la influencia de lenguas como el finés, el estonio y el vasco. Con la elección de dichos rasgos, Nolan pretende aportar a su invención lingüística una morfología rica, que le permita una mayor libertad en el orden de palabras.

Por otro lado, la forma en imperativo incluye también el pronombre, como ['sarhafassi]. Mientras que en alemán debe aparecer el pronombre de sujeto, en español puede omitirse: sagen Sie mir > (dígame usted). En el siguiente ejemplo proporcionado por Nolan (en 2015), encontramos esos casos del imperativo:

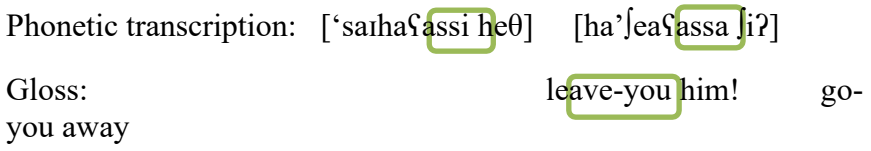

En este ejemplo aparece uno de los rasgos principales de este sistema; se trata de la diferencia en la terminación entre ['sarhąassi] y ['sarha@assa]. Este hecho implica que estamos ante una lengua ergativo-absolutiva. En estas, el sujeto de las construcciones intransitivas se marca de la misma forma que el objeto de las transitivas, y el sujeto de las transitivas se marca en una forma diferente. El sujeto de un transitivo corresponde al caso ergativo y tanto el objeto directo del transitivo como el sujeto del intransitivo se encuentran en caso absolutivo. Posiblemente, en este aspecto, Nolan tomó como referencia al euskera, como lengua natural, y al Ithkuil de Quijada, como lengua artificial, ya que ambas constituyen ejemplos de este tipo.

Por último, conviene apuntar que resulta sumamente complicado trazar un análisis del léxico inventado para este proyecto lingüístico, debido a que apenas hay muestras de vocabulario.

\section{CONCLUSIÓN}

Las lenguas artificiales creadas con propósitos artísticos desempeñan diversas funciones en los argumentos de ficción literaria o cinematográfica en los que se incorporan. En estos universos imaginarios, estos proyectos lingüísticos contribuyen a la construcción de entidades individuales o grupales gracias a 
la relación que guardan con las culturas que los sustentan y a que funcionan de manera idéntica a como lo hace una lengua natural en el mundo real. En los filmes de la saga Harry Potter, el pársel asocia a uno de los personajes principales de la trama, lo que refuerza la caracterización negativa de este $y$, a su vez, ayuda a la configuración de un entramado cultural estrechamente vinculado a las serpientes.

Con respecto a su método de construcción, Nolan se basa principalmente en la fonética para elaborar este proyecto lingüístico. La elección de sonidos fricativos y faríngeos, así como la incorporación de geminadas y dígrafos, responde a la necesidad de conseguir un efecto sonoro semejante al que emiten los reptiles. Por otro lado, el autor emplea rasgos morfosintácticos poco frecuentes en los idiomas europeos con el propósito de diferenciar este esquema lingüístico de las lenguas naturales. Con todo, nuestro análisis lingüístico sobre el pársel concluye en estas líneas, que esperamos retomar en futuras investigaciones.

\section{REFERENCIAS BIBLIOGRÁFICAS}

Anatol, Gizelle L. 2013. The Fallen Empire: Exploring Ethnic Otherness in the World of Harry Potter. En Gizelle L. Anatol (ed.). Reading Harry Potter: critical essays. Londres: Praeger.

Barnes, Lawrie y Chantelle van Heerden. 2006. Virtual languages in science fiction and fantasy literature. Language Matters 37 (1): 102-117.

Calero Vaquera, María Luisa. 1999. Proyectos de lengua universal. La contribución española. Córdoba: Publicaciones de la Universidad de Córdoba y Obra Social y Cultural Cajasur.

2010. Las irregularidades lingüísticas desde la perspectiva de los inventores de lenguas universales. En Carsten Sinner y Alfonso Zamorano (eds.). La excepción en la gramática española. Perspectivas de análisis. Lingüística Iberoamericana 41, pp. 75-34. Madrid y Frankfurt, Iberoamericana Editorial Vervuert.

Couturat, Louis y LÉOPOLd LeAu. 1903. Histoire de la langue universelle. Hildesheim: Georg Olms Verlag.

Galán Rodríguez, CARMen. 2018a. Género, sexo y lenguas artificiales. BSEHL 12: 75-93. 2017. La invención de lenguas musicales en la literatura de viajes fantásticos del siglo XVII. Revista argentina de historiografia lingüistica IX (1): 39-57.

2007. Logomaquias y logofilias: distopías linguisticas en la ficción literaria. Anuario de Estudios Filológicos XXX: 115-129.

GÁNDARA FernáNDEZ, LETICIA. 2019. Un análisis lingüístico de la lengua Klingon (Star Trek). Tonos digital: Revista de estudios filológicos 37: 1-16.

2016. Una lengua para mujeres: S. Haden Elgin y la creación del láadan. En Antonio Salvador et al. (eds.). La Historiografia Lingüística como paradigma de investigación, pp. 381-392. Madrid: Akal. 
Hewlett, Nigel y Janel M. Beck. 2006. An introduction to the science of phonetics. Mahwah: Lawrence Erlbaum.

Hilsheimer von Tessa. 2011. Word Magic: Defining Harry Potter's World in New Terms. Master of Arts in English Studies. Faculty of the Department of English. East Carolina University: East Carolina, USA

Karlsson, Fred. 1991. Gramática básica del finés [trad. Ursula Ojanen et al.]. Madrid: Ediciones de la Universidad Autónoma de Madrid.

Llisterri, Johquim. 2018. Métodos de análisis acústico del habla [en línea]. Disponible en http://liceu.uab.es/ joaquim/phonetics/fon_produccio/clasificacion_articulatoria,html [Consulta 25/01/2018].

Martínez Gavilán, María Dolores. 2016. La contribución de Caramuel a la creación de lenguas artificiales: características universales, lenguas filosóficas y lenguas secretas. Revista de Investigación Lingüística 19: 77-106.

OKrand, Marc. 1992. The Klingon Dictionary. English/Klingon, Klingon/English. The official guide to Klingon words and phrases. Nueva York: Pocket Books.

Olivera, DANiEl M. 2015. El arte élfico de la lingüística. Tolkien y su relación con el lenguaje y las lenguas artificiales. Estel 84: 51-66.

Peterson, David J. 2014. Living Language Dothraki. Nueva York: IVY Books.

Robles, José María. 2016. Así se crearon los idiomas de "Juego de Tronos", "Star Trek" y “Avatar". El Mundo [en línea]. Disponible en http://www.elmundo.es/papel/pantallas/20 15/12/03/5660187246163f92368b457d.html [Consulta 26/02/2018].

Rowling, JoAnne K. 1998 [2001]. Harry Potter y la piedra filosofal. Barcelona: Salamandra. 1999 [2001]. Harry Potter y la cámara secreta. Barcelona: Salamandra. 2001. Harry Potter y el cáliz de fuego. Barcelona: Salamandra.

Shippeys, Tom. 2000. J. R. R. Tolkien: Author of the Century. Londres: HarperCollins.

Tolkien, John R. R. 1993[1981]. Las cartas de J. R. R. Tolkien. Trad. de Rubén Masera. Barcelona: Minotauro. 